\title{
A novel NF1 mutation in a Chinese patient with giant café-au-lait macule in neurofibromatosis type 1 associated with a malignant peripheral nerve sheath tumor and bone abnormality
}

\author{
H.-X. Tong ${ }^{1 *}$, M. Li ${ }^{2 *}$, Y. Zhang ${ }^{1}, J$ Zhu ${ }^{1}$ and W.-Q. Lu ${ }^{1}$ \\ ${ }^{1}$ Department of General Surgery, Zhong Shan Hospital, Fu Dan University, \\ Shanghai, China \\ ${ }^{2}$ Department of Dermatology, Xinhua Hospital, School of Medicine, \\ Shanghai Jiaotong University, Shanghai, China \\ *These authors contributed equally to this study. \\ Corresponding author: W.-Q. Lu \\ E-mail: luweiqi6@gmail.com
}

Genet. Mol. Res. 11 (3): 2972-2978 (2012)

Received September 28, 2011

Accepted February 1, 2012

Published July 10, 2012

DOI http://dx.doi.org/10.4238/2012.July.10.6

\begin{abstract}
Neurofibromatosis type 1 (NF1; OMIM\#162200) is a common neurocutaneous disorder that is characterized by multiple café-au-lait, skinfold freckling, Lisch nodules, and neurofibromas. Mutations in the NF1 gene, which encodes the neurofibromin protein, have been identified as the pathogenic gene of NF1. In this study, we present a clinical and molecular study of a Chinese patient with giant café-aulait in NF1. The patient showed $>6$ café-au-lait spots on the body, axillary freckling, and multiple subcutaneous neurofibromas. He also had a malignant peripheral nerve sheath tumor and bone abnormalities. The germline mutational analysis of the NF1 gene revealed a novel missense mutation in exon 13. It is a novel heterozygous nucleotide $\mathrm{G}>\mathrm{A}$ transition at position 2241 of the NF1 gene. We found no mutation in malignant peripheral nerve sheath tumor DNA from this patient. This
\end{abstract}


expands the database for NF1 gene mutations in NF1. Its absence in the normal chromosomes suggests that it is responsible for the NF1 phenotype. To our knowledge, this is the first case of giant café-au-lait macule in NF1 associated with a malignant peripheral nerve sheath tumor and bone abnormality.

Key words: Mutation analysis; $N F 1$ gene; Neurofibromatosis type 1; Giant café-au-lait

\section{INTRODUCTION}

Neurofibromatosis type 1 (NF1; OMIM\#162200), also known as von Recklinghausen neurofibromatosis, is a common inherited disease and has a high rate of penetrance approaching $100 \%$ by age 20 (Huson et al., 1988). The incidence of this disease has been estimated to be 1 in 3500 individuals (Rasmussen and Friedman, 2000). It is characterized by café-aulait (CAL) spots, cutaneous or subcutaneous neurofibromas, freckling of axillary or inguinal regions, bone dysplasias and Lisch nodules of the iris (Gutmann et al., 1997). Further manifestations occur less frequently among patients with NF1, including malignancies, plexiform neurofibromas, optic glioma, learning difficulties, and juvenile xanthogranuloma. Skeletal abnormalities in NF1 are variable and include scoliosis, long bone or sphenoid dysplasia, macrocephaly, prominent brow, short stature, pectus excavatum, and pseudoarthrosis (especially of the tibia) (Gutmann et al., 1997). CAL spots occur in more than $90 \%$ of NF1 cases (Yang et al., 2008). They appear as well-circumscribed, light-brown, homogenous patches that range in size from 1 to $2 \mathrm{~mm}$ to greater than $20 \mathrm{~cm}$ in diameter, with the majority being under $10 \mathrm{~cm}$. Giant café-au-lait macules larger than $30 \mathrm{~cm}$ are rather unusual (Thappa et al., 2001; Erdi et al., 1999; Shah, 2010).

NF1 is caused by mutations of the NF1 gene on chromosome 17q11.2. This gene spans $350 \mathrm{~kb}$ of genomic DNA and contains 60 exons. It is composed of 2818 amino acid residues which encode neurofibromin (Bausch et al., 2007). Neurofibromin is widely expressed in many tissues and acts as a Ras GTPase-activating protein (GAP) (Brems et al., 2009). To date, more than 800 independent mutations in the NF1 gene among different races have been described to be associated with NF1 (Heim et al., 1995; Upadhyaya et al., 1997; Origone et al., 2002; Cai et al., 2005; Bausch et al., 2007; Upadhyaya et al., 2008; Bottillo et al., 2009; Messiaen et al., 2011). In this study, we performed germline and somatic mutation detection of the NF1 gene in a Chinese patient with giant café-au-lait macule in NF1-associated malignant peripheral nerve sheath tumours (MPNST) and bone abnormalities by sequencing and found a novel missense mutation.

\section{MATERIAL AND METHODS}

\section{Patient}

The patient was a 17-year-old Chinese boy who was followed up in our hospital because of pain of the left leg. He felt pain of the left leg three months prior and found an inferior abdominal mass twenty days prior himself. Clinical features consistent with a diagnosis of NF1 included CAL 
spots, axillary freckling and multiple subcutaneous neurofibromas. He presented with CAL spots on almost all of the body, with a diameter of $0.3-2.5 \mathrm{~cm}$, freckling on the axillary region, multiple subcutaneous neurofibromas and bone abnormality (Figure 1a and b). Another prominent feature was a giant café-au-lait macule with irregular borders, involving the lower part of the back, right flank and both buttocks and thighs (Figure 1c, $\mathrm{d}$ and e). None of his family members suffered from NF1. A mass on the right lower extremity of the tibia was resected at another hospital when he was 10 years old (details are unknown). He had lost weight, three kilograms in the preceding three months. Abdominal computed tomography (CT) showed a well-defined $10 \times 15 \mathrm{~cm}$ mass in the retroperitoneum (Figure 2a). X-rays showed a bone cyst within the metaphysis of the right tibial and congenital talipes equino varus (Figure $2 \mathrm{~b}$ ). CT and MRI showed a multiloculated cystic mass in the sacral canal and expanded sacral foramina. The diagnosis was right sacral foramina multiple nerve sheath cyst (Figure 2c and d). Surgical resection the tumor mass was performed. Histopathologic examination revealed MPNST, and he received radiotherapy (50 Gy) to the primary tumor.

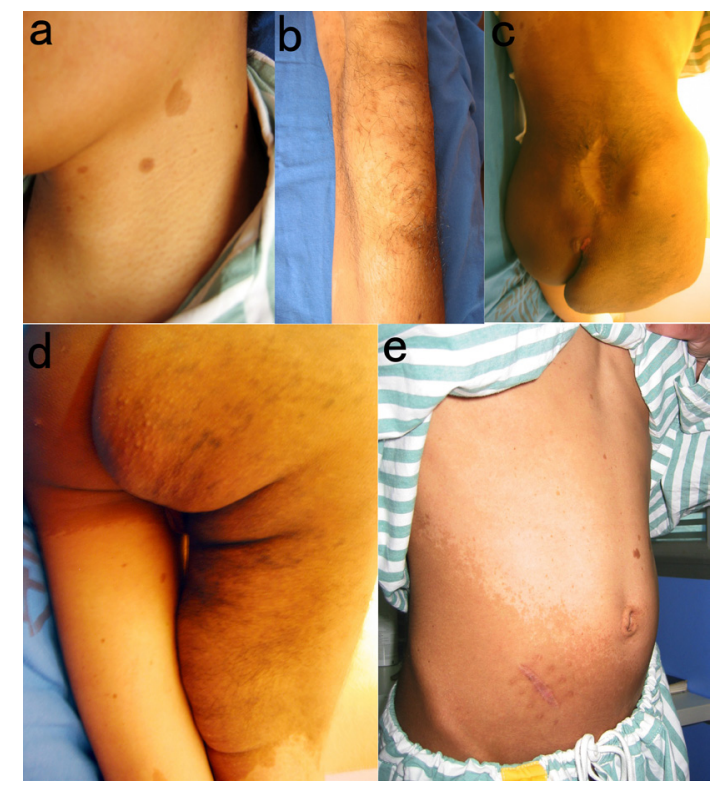

Figure 1. Clinical manifestations of the patient. a. Several tiny CALS on the left neck. b. Large subcutaneous neurofibroma on the right leg. c. d. Giant café-au-lait macule on the lower part of the back and both buttocks. e. Giant café-au-lait macule on the right flank.

\section{Mutational analysis}

The study protocol was approved by Ethical Committee of Zhongshan Hospital, Fu Dan University. After informed consent, genomic DNA was extracted from the patient's peripheral blood lymphocytes. We designed primers flanking all 60 coding exons and intron-exon boundaries of the NF1 gene using the web-based version of the Primer 3.0 program (http:// www.genome.wi.mit.edu/cgi-bin/primer/primer3_www.cgi). PCR was performed in a $15-\mu \mathrm{L}$ reaction volume containing $20 \mathrm{ng}$ genomic DNA, $0.3 \mathrm{mM}$ dNTPs, $0.3 \mu \mathrm{M}$ of each primer, 3.0 
$\mathrm{mM} \mathrm{MgCl}{ }_{2}$ and $0.1 \mathrm{U}$ Taq DNA polymerase. The PCR conditions were: Taq activation at $95^{\circ} \mathrm{C}$ for $15 \mathrm{~min}$, followed by 40 cycles of denaturation at $94^{\circ} \mathrm{C}$ for $40 \mathrm{~s}$, annealing at $58^{\circ} \mathrm{C}$ for 60 $\mathrm{s}$ and extension at $72^{\circ} \mathrm{C}$ for $55 \mathrm{~s}$, except that in the first ten cycles the annealing temperature decreased from $63^{\circ}$ to $58^{\circ}$ by $0.5^{\circ} \mathrm{C}$ per cycle; and a final extension was at $72^{\circ} \mathrm{C}$ for $10 \mathrm{~min}$. After the amplification, the products were purified using a QIAquick PCR Purification kit (Qiagen). We sequenced the NF1 gene using an ABI PRISM ${ }^{\circledR} 3730$ automated sequencer (Applied Biosystems). Sequence comparisons and analysis were performed using Phred-PhrapConsed Version 12.0 program. In addition, samples from 110 unrelated population-match controls were sequenced for missense to exclude the possibility that these were polymorphisms in the NF1 gene. Mutations were identified by comparing with the reported cDNA reference sequence (GenBank accession No.: NM_000267.1). A multiplex ligation-dependent probe amplification (MLPA) analysis identified small deletions or duplications involving adjacent exons (NF1 gene-specific MLPA Assay kits P081/082 and P122, MRC Holland, Amsterdam, NL) as previously reported (Upadhyaya et al., 1997).

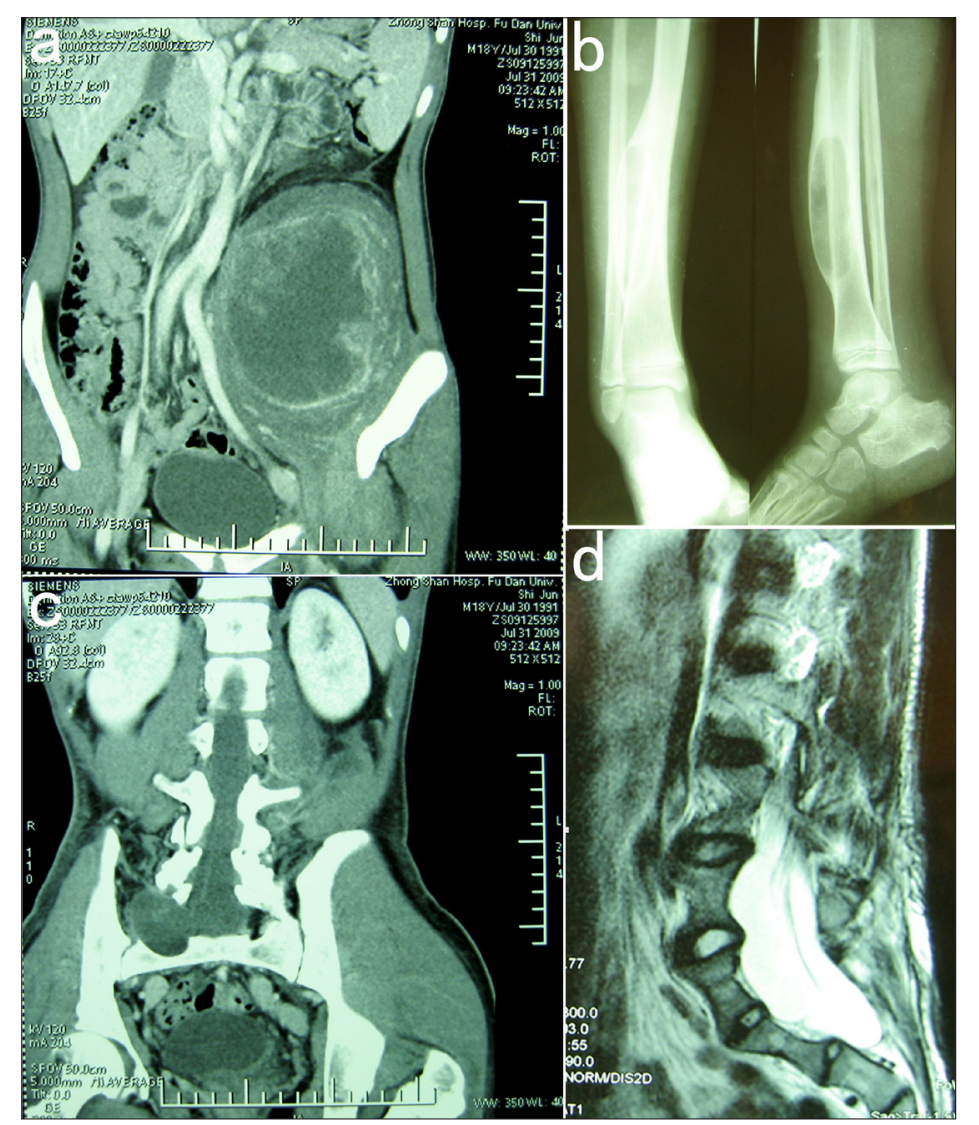

Figure 2. Radiologic images of the patient. a. Abdominal computed tomography (CT) showed a well-defined 10 $\mathrm{x} 15 \mathrm{~cm}$ mass in the retroperitoneum. b. X-ray showed a bone cyst within the metaphysis of the right tibial and congenital talipes equino varus. c. d. CT and MRI showed a multiloculated cystic mass in the sacral canal and expanded sacral foramina. 


\section{RESULTS}

Direct DNA sequencing showed a novel heterozygous missense mutation (c.2241 $\mathrm{G}>\mathrm{T}$ ) in exon 13 of NF1 gene. This mutation results in replacement of methionine (ATG) by isoleucine (ATA) (Figure $3 \mathrm{a}$ and b). It was not found in any of the 110 healthy controls showing that it is a novel pathogenic mutation, not a common polymorphism. We found no mutation in MPNST DNA from this patient.

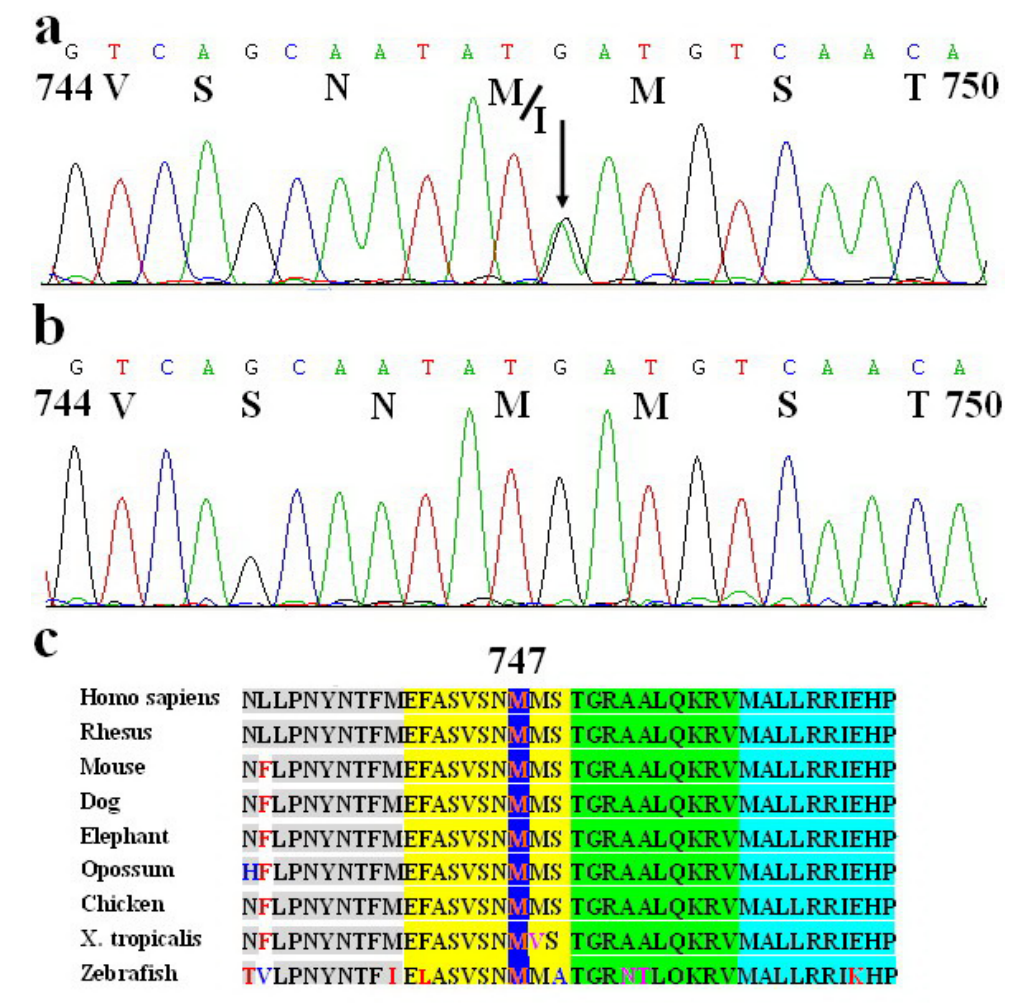

Figure 3. Mutant sequence and wild type sequence and amino acid alignment of various species around the residue 747 of NF1. a. Partial DNA sequence of exon 13 of $N F 1$ gene (wild type). b. The heterozygous mutation c. $2822 \mathrm{G}>\mathrm{T}$ in the DNA sample of patient. The black arrow indicates the mutation. c. Residue M at 747 is conserved in all species compared in the figure. All data were cited from GenBank.

\section{DISCUSSION}

Here, we report on a boy with typical lesions of neurofibromatosis type 1 . He also had a giant CAL in the form of a garment-like pigmented area with serrated margins. Giant CAL exceeding $30 \mathrm{~cm}$ in diameter is rare (Yang et al., 2008). Yang et al. (2008) reported a case with a giant CAL in neurofibromatosis type 1 . They speculated that large CAL may be explained as an example of type 2 segmental NF1. The giant CAL is supposed to be caused by loss of the corresponding wild type allele of the NF1 gene (Yang et al., 2008). To the best of our knowl- 
edge, there have been no documented case reports of an NF1 patient with giant CAL, MPNST and bone abnormality to date.

The main function of neurofibromin is downregulating the biological activity of the proto-oncoprotein Ras by acting as a Ras-specific GTPase activating protein. NF1 protein has two functional domains (Brems et al., 2009). The first is the GAP-related domain, which downregulates Ras activity and is encoded by exons 21-27a. The second region is a Cys-Ser rich domain. This domain, which is encoded by exons 11-17, has three cysteine pairs suggestive of ATP binding as well as three potential cAMP-dependent protein kinase recognition sites (Cichowski and Jacks, 2001). In the present study, the novel $2241 \mathrm{G} \rightarrow \mathrm{A}$ mutaion was found in a Chinese NF1 patient, whicht had resulted in p.M747I in exon13. The amino acid residue at 747 located the second functional domain, which is suspected to play an important role in phosphorylation, and the mutation at this position could probably influence its function. Therefore, it is possible that this mutated allele encodes a dysfunctional neurofibromin. The loss of or diminished function of the protein, leads to accumulation of the activated Ras, and the process of suppressing cell growth is left unhindered. However, NF1 protein was not analyzed in this patient. It is noteworthy that the amino acid residue at 747 is highly conserved in different species, including human, mouse, rat, frog, zebrafish, and puffer fish (Figure 3c).

Patients with NF1 are at greatest risk for bone abnormalities. This patient had long bone dysplasia and spina bifida. The long bone dysplasia and spinal deformities are very common in patients with NF1. The underlying pathogenic mechanisms of neural tube defects are not fully understood and have a multifactorial etiology. In a Splotch mouse model, Nf1 is important for the development of neural crest-derived structures and the central nervous system. Neural development is grossly normal in heterozygotes, where neural tube defects are not seen. Nf1(-/-) homozygous mutant embryos develop spina bifida and sometimes exencephaly. The experimental evidence suggests that osteoblasts are deficient and their osteoclasts have increased survival rates, leading to increased degradation of bony tissue (Lakkis et al., 1999). Recently, Zhang et al. (2011) showed that Nf1flox/-; Col2.3Cre+ mice display multiple structural and functional abnormalities in the lumbar vertebrae. The murine model displays reduced bone mass, increased osteoclast formation and reduced osteoblast numbers in the vertebrae (Zhang et al., 2011). These results supported the idea that NF1 gene mutation may be the cause of multiple spinal deformities in NF1 patients.

Patients with NF1 are at greatest risk for developing sarcomas, including MPNST (Ferner and Gutmann, 2002; Kar et al., 2006; Bottillo et al., 2009). It is an uncommon neoplasm that develops within a peripheral nerve. The incidence of MPNST arising in NF1 is $4.6 \%$, which is much higher than the $0.001 \%$ rate in the general population (Evans et al., 2002). To date, the pathogenesis of NF1 associated with MPNST remains unclear. The germline mutations of patients with NF1 and MPNST include missense, nonsense, frameshift, splicing abnormalities, and a large genomic deletion (Upadhyaya et al., 2008; Bottillo et al; 2009). Somatic NF1 mutations found in MPNST DNA samples are mostly large genomic deletions that partially or completely delete the NF1 gene. Upadhyaya et al. (2008) found that NF1 patients with a small germline mutation are more likely to develop large somatic deletions in any MPNST tumors that develop. However, we could not find any mutation of the NF1 gene in MPNST DNA from this patient.

In conclusion, we identified a novel germline mutation of the $N F 1$ gene in a Chinese patient with a giant CAL in NF1-associated MPNST and bone abnormality. We enlarged the 
spectrum of mutations in the NF1 gene. The ongoing recognition of different mutations may give insight into the still unknown mechanisms involved in the development of NF1.

\section{ACKNOWLEDGMENTS}

Research supported by the Youth Foundation of Zhongshan Hospital, Fudan University (\#201101) and Shanghai Municipal Natural Science Foundation (\#12ZR1420000). We are most grateful to the patient for participating in this study.

\section{REFERENCES}

Bausch B, Borozdin W, Mautner VF, Hoffmann MM, et al. (2007). Germline NF1 mutational spectra and loss-ofheterozygosity analyses in patients with pheochromocytoma and neurofibromatosis type 1. J. Clin. Endocrinol. Metab. 92: 2784-2792.

Bottillo I, Ahlquist T, Brekke H, Danielsen SA, et al. (2009). Germline and somatic NF1 mutations in sporadic and NF1associated malignant peripheral nerve sheath tumours. J. Pathol. 217: 693-701.

Brems H, Beert E, de RT and Legius E (2009). Mechanisms in the pathogenesis of malignant tumours in neurofibromatosis type 1. Lancet Oncol. 10: 508-515.

Cai Y, Fan Z, Liu Q, Li J, et al. (2005). Two novel mutations of the NF1 gene in Chinese Han families with type 1 neurofibromatosis. J. Dermatol. Sci. 39: 125-127.

Cichowski K and Jacks T (2001). NF1 tumor suppressor gene function: narrowing the GAP. Cell 104: 593-604.

Erdi H, Boyvat A and Calikoglu E (1999). Giant cafe au lait spot in a patient with neurofibromatosis. Acta Derm. Venereol. 79: 496.

Evans DG, Baser ME, McGaughran J, Sharif S, et al. (2002). Malignant peripheral nerve sheath tumours in neurofibromatosis 1. J. Med. Genet. 39: 311-314.

Ferner RE and Gutmann DH (2002). International consensus statement on malignant peripheral nerve sheath tumors in neurofibromatosis. Cancer Res. 62: 1573-1577.

Gutmann DH, Aylsworth A, Carey JC, Korf B, et al. (1997). The diagnostic evaluation and multidisciplinary management of neurofibromatosis 1 and neurofibromatosis 2. JAMA 278: 51-57.

Heim RA, Kam-Morgan LN, Binnie CG, Corns DD, et al. (1995). Distribution of 13 truncating mutations in the neurofibromatosis 1 gene. Hum. Mol. Genet. 4: 975-981.

Huson SM, Harper PS and Compston DA (1988). Von Recklinghausen neurofibromatosis. A clinical and population study in south-east Wales. Brain 111: 1355-1381.

Kar M, Deo SV, Shukla NK, Malik A, et al. (2006). Malignant peripheral nerve sheath tumors (MPNST)-clinicopathological study and treatment outcome of twenty-four cases. World J. Surg. Oncol. 4: 55.

Lakkis MM, Golden JA, O'Shea KS and Epstein JA (1999). Neurofibromin deficiency in mice causes exencephaly and is a modifier for Splotch neural tube defects. Dev. Biol. 212: 80-92.

Messiaen L, Vogt J, Bengesser K, Fu C, et al. (2011). Mosaic type-1 NF1 microdeletions as a cause of both generalized and segmental neurofibromatosis type-1 (NF1). Hum. Mutat. 32: 213-219.

Origone P, De LA, Bellini C, Buccino A, et al. (2002). Ten novel mutations in the human neurofibromatosis type 1 (NF1) gene in Italian patients. Hum. Mutat. 20: 74-75.

Rasmussen SA and Friedman JM (2000). NF1 gene and neurofibromatosis 1. Am. J. Epidemiol. 151: 33-40.

Shah KN (2010). The diagnostic and clinical significance of cafe-au-lait macules. Pediatr. Clin. North Am. 57: 1131-1153.

Thappa DM, Jeevankumar B and Karthikeyan K (2001). Giant cafe-au-lait macule in neurofibromatosis type 1. J. Dermatol. 28: 60-61.

Upadhyaya M, Osborn MJ, Maynard J, Kim MR, et al. (1997). Mutational and functional analysis of the neurofibromatosis type $1(N F 1)$ gene. Hum. Genet. 99: 88-92.

Upadhyaya M, Kluwe L, Spurlock G, Monem B, et al. (2008). Germline and somatic NF1 gene mutation spectrum in NF1associated malignant peripheral nerve sheath tumors (MPNSTs). Hum. Mutat. 29: 74-82.

Yang CC, Happle R, Chao SC, Yu-Yun LJ, et al. (2008). Giant cafe-au-lait macule in neurofibromatosis 1: a type 2 segmental manifestation of neurofibromatosis 1? J. Am. Acad. Dermatol. 58: 493-497.

Zhang W, Rhodes SD, Zhao L, He Y, et al. (2011). Primary osteopathy of vertebrae in a neurofibromatosis type 1 murine model. Bone 48: 1378-1387. 